\title{
Germanium Based Two-Dimensional Photonic Crystals: The Hexagonal and Honeycomb Lattices
}

\section{Fairuz Aniqa Salwa, Muhammad Mominur Rahman, Muhammad Obaidur Rahman, Muhammad Abdul Mannan Chowdhury}

Department of Physics, Jahangirnagar University, Dhaka, Bangladesh

Email: mmrahman.phy@gmail.com

How to cite this paper: Salwa, F.A., Rahman, M.M., Rahman, M.O. and Chowdhury, M.A.M. (2019) Germanium Based Two-Dimensional Photonic Crystals: The Hexagonal and Honeycomb Lattices. Optics and Photonics Journal, 9, 25-36.

https://doi.org/10.4236/opj.2019.93004

Received: February 1, 2019

Accepted: March 26, 2019

Published: March 29, 2019

Copyright $\odot 2019$ by author(s) and Scientific Research Publishing Inc. This work is licensed under the Creative Commons Attribution International License (CC BY 4.0).

http://creativecommons.org/licenses/by/4.0/

\begin{abstract}
The properties of two-dimensional (2D) photonic crystals (PCs) composed of germanium $(\mathrm{Ge})$ are discussed. We investigate polarization-dependent photonic band diagrams (transverse electric and transverse magnetic polarizations), gap maps, surface plots, contour maps, etc. for 2D PCs with Ge rods in air and vice versa for two different lattices geometries, namely hexagonal and honeycomb lattices. The obtained graphs for the four possible combinations are presented in this paper. All the graphs depict clear photonic band gaps. The conditions for the largest TE and TM band gaps are described. The honeycomb lattice of Ge rods in air background offers a large complete photonic band gap $\Delta \omega / \omega_{m}$ greater than $8 \%$ (for rod radius of $r=0.2 \mu \mathrm{m}$ ). Using these data, new Ge based photonic devices can be fabricated to confine, control and manipulate light in a more useful way.
\end{abstract}

\section{Keywords}

Photonic Crystals, Transverse Electric Modes, Transverse Magnetic Modes, Photonic Band Diagram, Gap Map, Hexagonal Lattice, Honeycomb Lattice

\section{Introduction}

Photonic crystals are one of the most talked of topics of the modern world of science and engineering. They are a new frontier of research in modern physics. Photonic crystals are novel type of optical media with periodic variation of the refractive index, with a period comparable to the wavelength of light [1] [2]. They control the propagation properties of light photons in the same way as the natural crystals modify the properties of electrons in solid state physics [3] [4]. 
The determination of the eigenfunctions in a PC is similar to the calculation of the particle wave functions in solid-state. This similarity is used to obtain photonic band diagram or photonic band structure [1] [5] [6].

PCs possess several interesting physical properties like inhibition to spontaneous emission [3], Anderson localization of photons [7] etc. The most significant property of PCs is the presence of photonic band gap (PBG): a forbidden frequency range in which light of specific wavelength cannot propagate [1] [5] [6] [8]. When light with frequency inside the PBG incidents on this crystal, it is completely reflected. Depending on the geometry of the structure, PCs can be divided into three broad categories, namely: one-dimensional (1D), two-dimensional (2D) and three-dimensional (3D) PCs. 1D PCs are periodic along one direction, 2D PCs are periodic along two directions and 3D PCs are periodic along three directions.

However, 2D PCs can have large variety of configurations in comparison to the 1D PCs and are much easier to fabricate than 3D PCs. Furthermore, 2D PCs have many important applications. For this reason, photonic band gap calculations for two-dimensional photonic crystals have been the subject of considerable attention nowadays [9]-[14]. The eigen-modes of two-dimensional (2D) photonic crystal must be separated into two types i.e. the polarizations decouple into two types: 1) Transverse electric polarization; and 2) Transverse magnetic polarization.

Transverse electric (TE) modes: TE modes have nonzero components of $H_{x}$, $H_{z}$ and $E_{y}$. The electric field vector $(E)$ is always perpendicular to the plane of incidence.

Transverse magnetic (TM) modes: TM modes have non-zero components of $H_{y}, E_{x}$ and $E_{z}$ In this case, the electric field vector $(E)$ is parallel to the plane of incidence.

The above given definitions of TE and TM polarizations are used in our software RSoft which are opposite to the conventions used in some photonic crystal books [15] [16] and papers [17]. Although there is a research paper on silicon based 2D PC [18], but, to our knowledge, there exists no publication on the properties of germanium based 2D PCs. In this paper, several properties of two-dimensional photonic crystals composed of germanium are studied. We consider four cases i.e. hexagonal lattice of germanium rods in air, hexagonal lattice of air holes in germanium, honeycomb lattice of germanium rods in air and honeycomb lattice of air holes in germanium. A detailed numerical study of the properties of these photonic crystals is performed by presenting various TE and TM photonic band diagrams, photonic band gaps, surface plots, equi-frequency plots, gap maps etc. Photonic band gap data are provided also for each and every combination.

\section{Theoretical Background}

The behavior of electromagnetic (EM) radiation in a PC is described by Maxwell's equations [19]. We consider a source free medium that has no electric or magnetic current sources. Then, assuming linear, isotropic, nondispersive, per- 
fect dielectric materials, Maxwell's equations govern to the "master equation" for PC [1] [6] [20]:

$$
\nabla \times\left[\frac{1}{\varepsilon(\boldsymbol{r})} \nabla \times \boldsymbol{H}(\boldsymbol{r})\right]=\frac{\omega^{2}}{c^{2}} \boldsymbol{H}(\boldsymbol{r}) .
$$

where, $\varepsilon(\boldsymbol{r})$ is a periodic dielectric function, $\boldsymbol{H}(\boldsymbol{r})$ is the magnetic field inside the PC, $\omega$ is the frequency of EM radiation and $c$ is the speed of light. Applying Bloch-Floquet theorem, the solutions of the master equation can be expressed as:

$$
\boldsymbol{H}_{\boldsymbol{k}}(\boldsymbol{r})=e^{i \boldsymbol{k} \cdot \boldsymbol{r}} \boldsymbol{u}_{\boldsymbol{k}}(\boldsymbol{r}) .
$$

where, $\boldsymbol{k}$ is the Bloch wave vector, $\boldsymbol{r}$ is the position vector, $\boldsymbol{u}_{\boldsymbol{k}}(\boldsymbol{r})$ is a periodic function of the lattice given by:

$$
u_{k}(r)=u_{k}(r+R)
$$

where, $R$ is the lattice vector.

In order to solve various problems of PCs, powerful computational techniques have been developed. Some of the well-known methods are plane wave expansion method (PWE) [21] [22], finite difference time domain method (FDTD) [20], finite difference frequency domain method (FDFD), finite element method (FEM) etc. Among these methods, we used the PWE in this paper as it is the easiest method.

\section{Results and Discussions}

\subsection{Hexagonal Lattice of Germanium Rods in Air}

We consider a two-dimensional hexagonal lattice of circular Ge rods embedded in air background as shown in Figure 1(a). The crystal is periodic along the $x z$-plane and homogeneous along the $y$-direction. The radius of the Ge cylinders is, $r=0.2 \mu \mathrm{m}$ as shown in the unit cell of the crystal lattice in Figure 1(b) and the lattice constant is, $a=1 \mu \mathrm{m}$. So, the relative radius [18] is, $\frac{r}{a}=0.2$. The filling factor which is the ratio of the total cross-sectional area of the rods to the area of the unit cell [23] is, $f=0.126$.

The Brillouin zone and the triangular irreducible Brillouin zone for this $2 \mathrm{D}$ hexagonal lattice are shown in Figure 1(c).

The surface plots for TE and TM polarizations for this structure are plotted in Figure 2(a) and Figure 2(b) respectively. We have the real view of the photonic band gaps from these plots. The photonic band diagrams and the gap map for this structure for both polarizations are shown in Figure 3(a) and Figure 3(b) respectively. From the surface plots and band diagrams, we observe that there are three TE band gaps and one TM band gap for this structure. The band gap between the first and second TE band is very large, but the TM band gap is small. Also, the TE and TM band gaps do not overlap with each other. So, we have no complete band gap for this structure. 


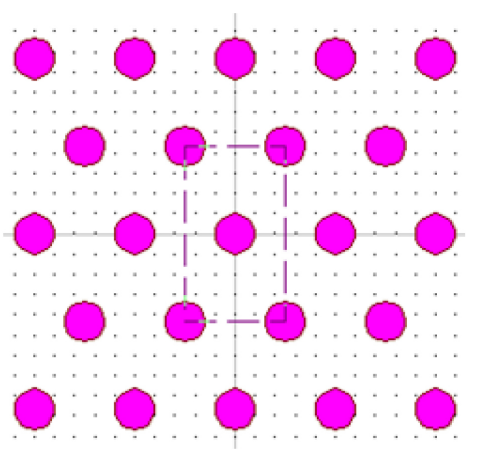

(a)

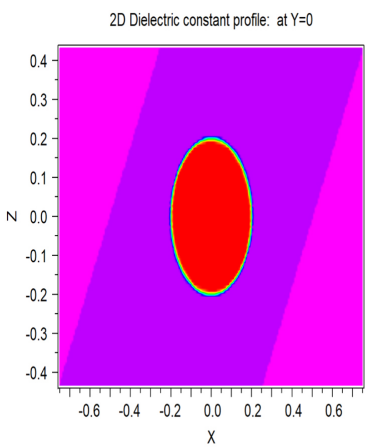

(b)

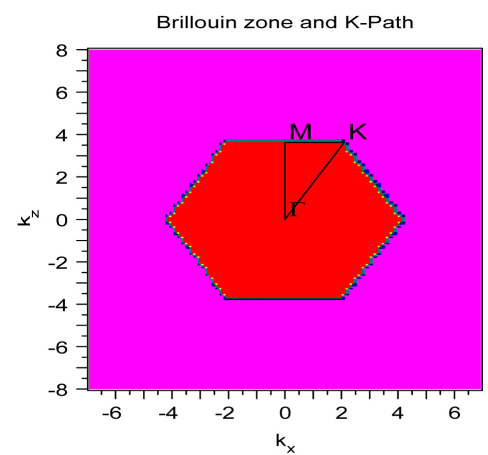

(c)

Figure 1. (a) A two-dimensional hexagonal lattice composed of germanium rods in air that was designed in the RSoft CAD layout; (b) A single unit cell of this lattice and (c) the hexagonal Brillouin zone with the irreducible Brillouin zone delimited by the three high-symmetry points $\Gamma, M$ and $K$.

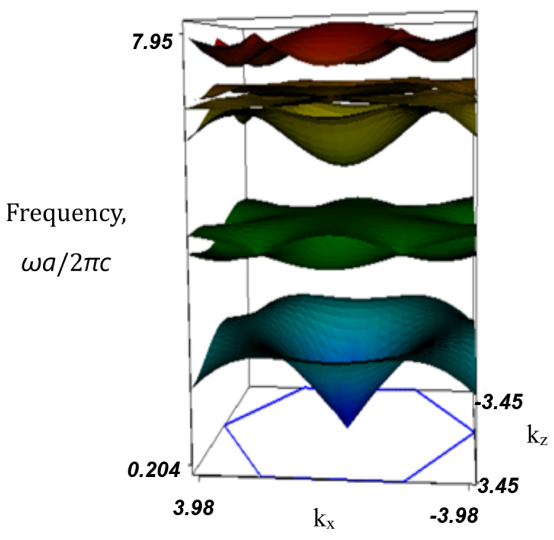

(a)

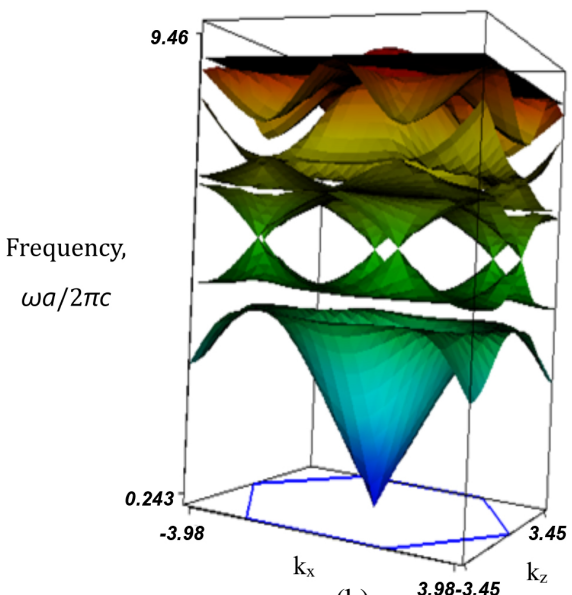

(b)

Figure 2. Surface plots for the two-dimensional hexagonal lattice composed of germanium rods in air for (a) TE-polarized and (b) TM polarized modes.

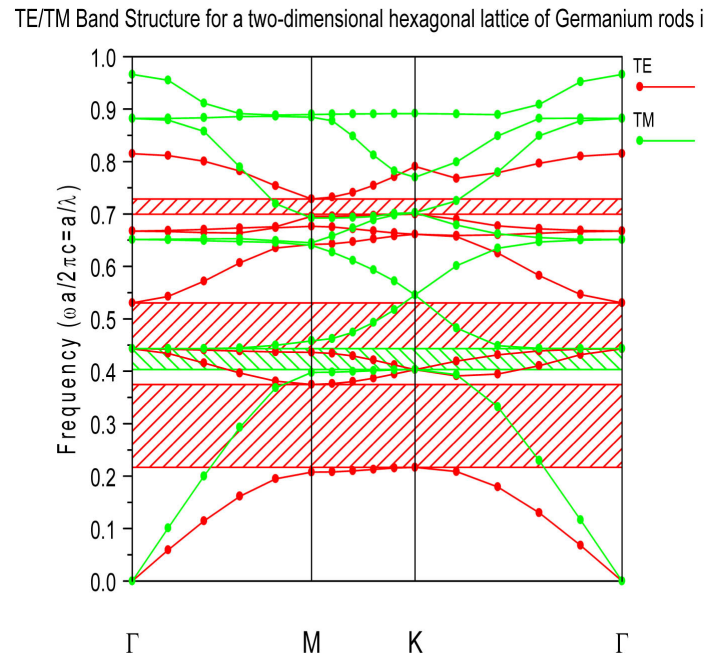

(a)

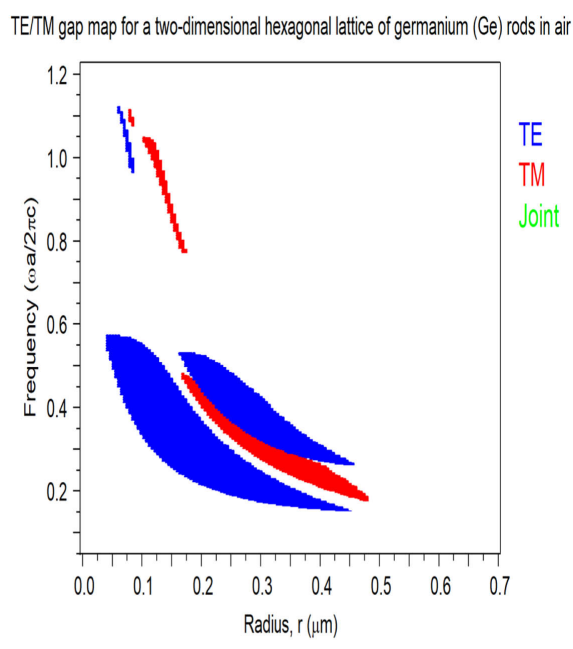

(b)

Figure 3. (a) The TE and TM both or hybrid band diagram for the two-dimensional hexagonal lattice of Ge rods in air and (b) the gap map for this structure. 
From the gap map of Figure 3(b), we observe that, as the radius of the rods is increased, a TE band-gap appears first at $r=0.037886 \mu \mathrm{m}$ and extends upto $r$ $=0.45687 \mu \mathrm{m}$. A TM band gap appears at $r=0.074485 \mu \mathrm{m}$ and extends upto $r$ $=0.4819 \mu \mathrm{m}$. There is no complete band gap for this material combination. The largest TE band-gap occurs at $r=0.119997 \mu \mathrm{m}$. The largest TM band-gap occurs at $r=0.1284 \mu \mathrm{m}$. At the radius of about $0.4 \mu \mathrm{m}$, there are two TE band gaps and one TM band gap. All the gaps seal up at the radius of about $0.5 \mu \mathrm{m}$, because at this radius value, the Ge rods begin to touch one another. There is no air gap between the Ge rods to open a photonic band gap. The band-gap width, mid-gap frequency, gap-midgap ratio and gap percentage for this structure are given in Table 1.

\subsection{Hexagonal Lattice of Air Holes in Germanium}

The crystal structure is reversed by inserting air holes in Ge background and the resulting band diagram for both polarizations for this structure is plotted in Figure 4(a). This figure shows that there is no band gap for TE polarization. Although a small gap opens for TM polarization.

The gap map for this structure for both polarizations is shown in Figure 4(b). From this map, we observe that, at first, a TM band gap appears for this structure at $r=0.17216 \mu \mathrm{m}$. Then a small TE band gap appears first at $r=0.22368$ $\mu \mathrm{m}$. A complete photonic band gap appears also for a radius of $r=0.40186 \mu \mathrm{m}$. The largest TE gap occurs at $r=0.5 \mu \mathrm{m}$. The largest TM gap occurs at $r=$ $0.46496 \mu \mathrm{m}$. The largest complete band gap for this structure occurs at $r=0.49$ $\mu \mathrm{m}$. Its width is $\Delta \omega=0.13969(2 \pi c / a)$. We see, a complete photonic band gap appears in this case, but for germanium rods in air we did not get a complete photonic band gap. The band-gap width, mid-gap frequency, gap-midgap ratio and gap percentage for this photonic crystal are given in Table 2.

\subsection{Honeycomb Lattice of Germanium Rods in Air}

Next we consider a two-dimensional honeycomb lattice of circular germanium (Ge) rods embedded in air background as shown in Figure 5(a). We consider the same radius value of the Ge rods i.e. $r=0.2 \mu \mathrm{m}$ as shown in the unit cell of Figure 5(b). The Brillouin zone and the triangular irreducible Brillouin zone for this $2 \mathrm{D}$ honeycomb lattice are shown in Figure 5(c).

The equifrequency plots for TE mode and TM mode for the considered structure

Table 1. TE and TM band gap data for the two-dimensional hexagonal lattice of Ge rods in air.

\begin{tabular}{cccccccc}
\hline $\begin{array}{c}\text { Crystal } \\
\text { structure }\end{array}$ & Polarization & Band & $\frac{\omega a}{2 \pi c}$ & $\begin{array}{c}\text { The mid-gap frequency } \\
\text { of the gap }\left(\omega_{m}\right)\end{array}$ & $\begin{array}{c}\text { Band-gap width } \\
(\Delta \omega)\end{array}$ & $\begin{array}{c}\text { Gap-midgap ratio Gap percentage } \\
\left(\Delta \omega / \omega_{m}\right)\end{array}$ \\
\hline $\begin{array}{c}\text { Hexagonal } \\
\text { lattice of }\end{array}$ & $\mathrm{TE}$ & $2^{\text {st }}$ & $0.21613-0.37472$ & 0.2957508839032 & 0.1582820154111 & 0.5351869564078 & 53.52 \\
$\begin{array}{c}\text { germanium } \\
\text { rods in air }\end{array}$ & & $3^{\text {sd }}$ & $0.69928-0.72882$ & 0.7141210658904 & 0.02931735176066 & 0.04105375567392 & 4.11 \\
& $\mathrm{TM}$ & $1^{\text {st }}$ & $0.40284-0.44292$ & 0.4233175504743 & 0.03957080196718 & 0.09347782042782 & 9.35 \\
\hline
\end{tabular}


Table 2. TM band gap data for the two-dimensional hexagonal lattice of air holes in germanium (Ge).

\begin{tabular}{cccccc}
\hline Crystal constituent & $\frac{\omega a}{2 \pi c}$ & $\begin{array}{c}\text { The mid-gap frequency } \\
\text { of the gap }\left(\omega_{m}\right)\end{array}$ & $\begin{array}{c}\text { Band-gap width } \\
(\Delta \omega)\end{array}$ & $\begin{array}{c}\text { Gap-midgap ratio } \\
\left(\Delta \omega / \omega_{m}\right)\end{array}$ & $\begin{array}{c}\text { Gap percentage } \\
(\%)\end{array}$ \\
\hline $\begin{array}{c}\text { Hexagonal lattice of air } \\
\text { holes in Ge }\end{array}$ & $0.15229-0.16426$ & 0.1586047011012 & 0.01260062381216 & 0.07944672336110 & 7.94 \\
\hline
\end{tabular}

TE/TM Band Diagram for a Two-Dimensional Hexagonal Lattice of Air Holes in Germanium

TETTM gap map for a two-dimensional hexagonal lattice of air holes in germanium (Ge)

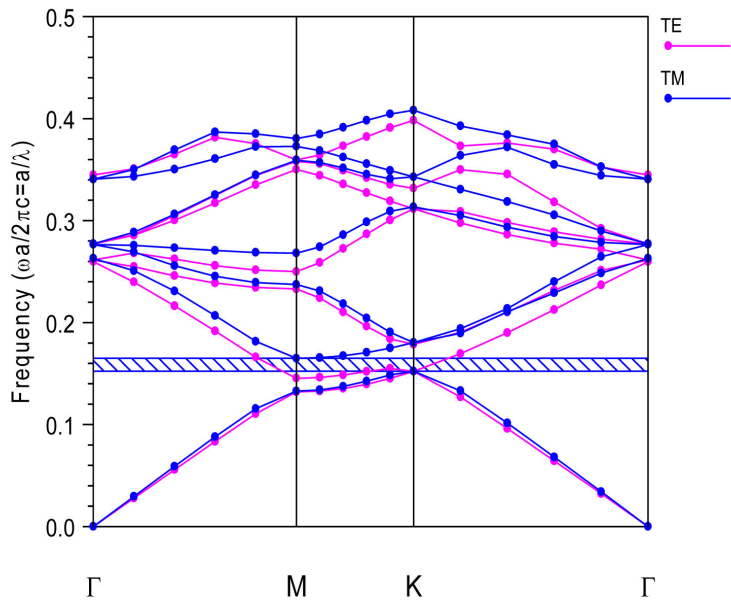

(a)

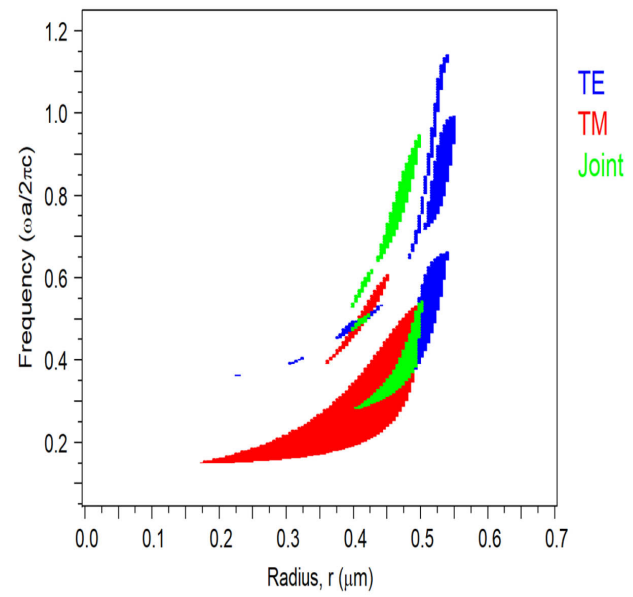

(b)

Figure 4. (a) The TE and TM both or hybrid band diagram for a two-dimensional hexagonal lattice of air holes in germanium and (b) the gap map for this structure.

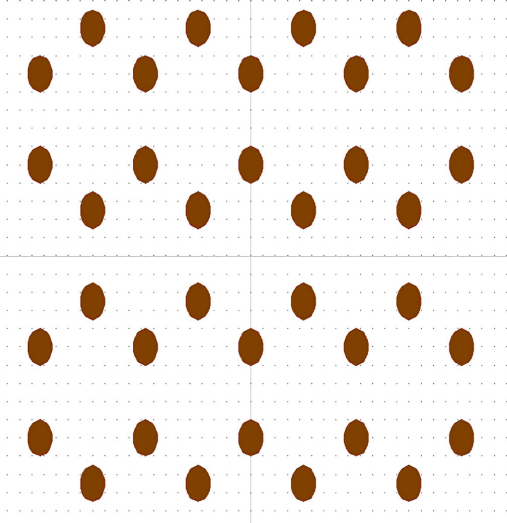

(a)

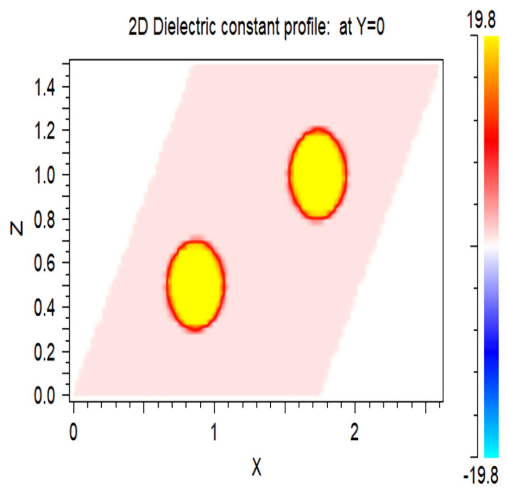

(b)

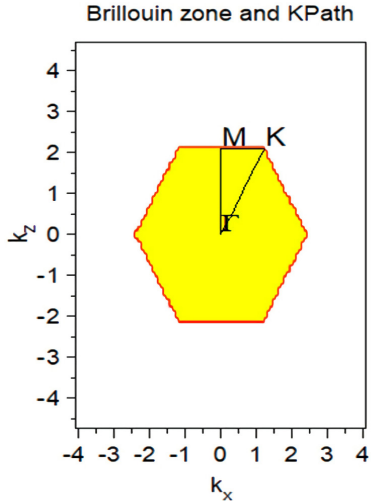

(c)

Figure 5. (a) The Rsoft CAD layout view of a two-dimensional honeycomb lattice composed of germanium rods in air; (b) A single unit cell of this lattice; (c) The Brillouin zone with the irreducible Brillouin zone marked by the three high-symmetry points $\Gamma, M$ and $K$.

are shown in Figure 6 and Figure 7 respectively. Equi-frequency or isofrequency contour is supplementary in analyzing behaviors of photonic crystals, which is the intersection of a constant frequency $\omega$-plane to a dispersion surface [24]. It quantifies all allowed wave vectors in the photonic crystals and their corresponding frequencies. The contour lines are called frequency isolines.

In the equi-frequency plots of Figure 6 and Figure 7, the first Brillouin zone 


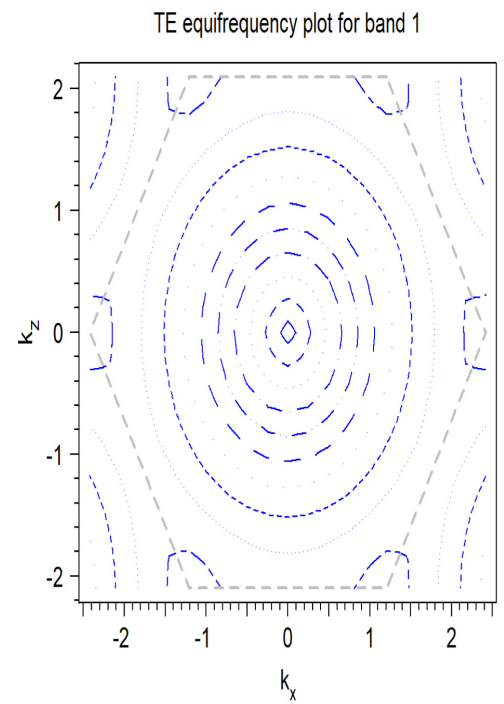

(a)

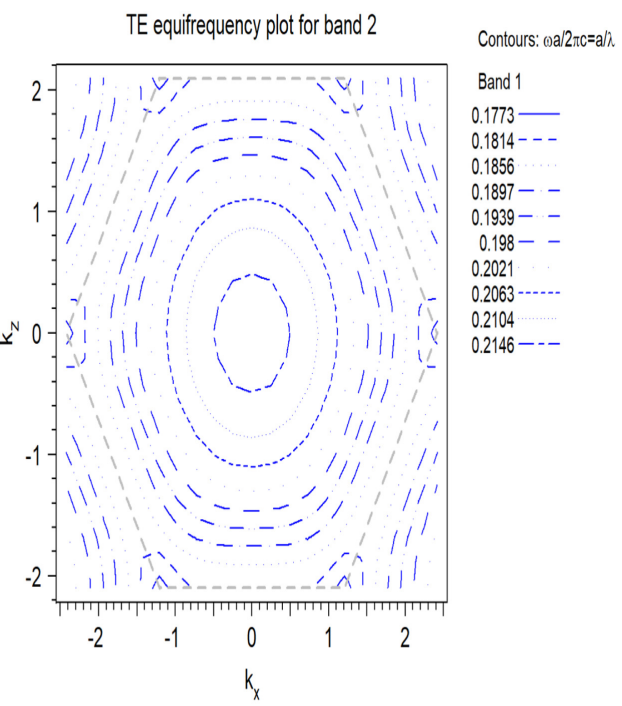

(b)

Figure 6. The equi-frequency plots for the first two TE bands for the two-dimensional honeycomb lattice of germanium rods in air.

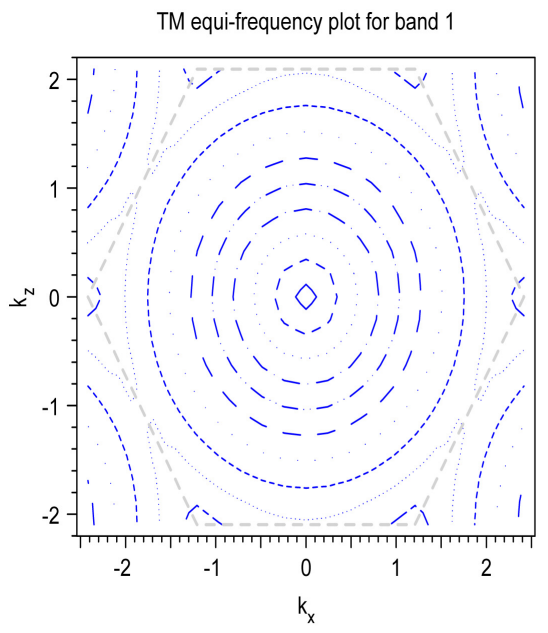

(a)
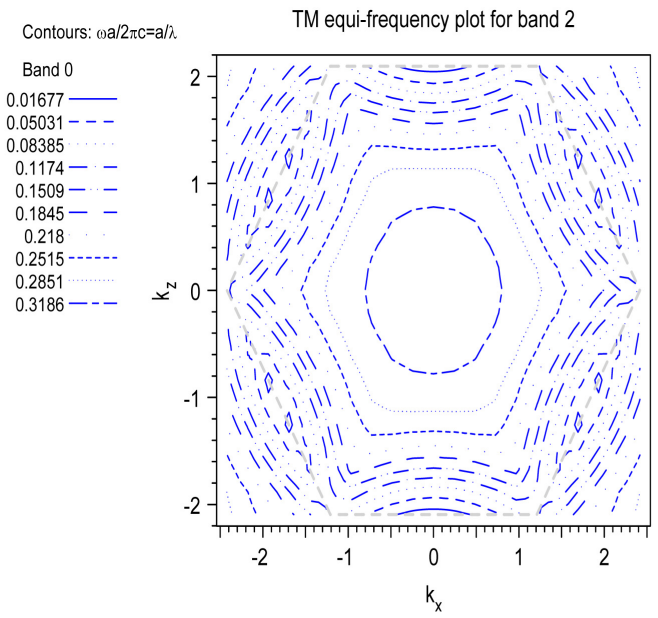

(b)

Figure 7. The equi-frequency plots for the first two TM band for the two-dimensional honeycomb lattice of germanium rods in air.

is indicated by the gray dashed lines. For the first and second TE bands, the shape of the frequency isolines is almost circular. For the first TM band, the shape of the frequency isolines is approximately circular also. However, for the second band, the shape of the frequency isolines is quite complex. For the first band, the frequency of light is small and wavelength is large. If the wavelength of light is increased than the size of the crystal unit cell, then the light reacts on the averaged permittivity. That is why the photonic crystal can be considered as a homogenous media in that case. When the wave vector $k$ is close to the boundary of the Brillouin zone and the wavelength is comparable with the size of the unit cell, the shape of the frequency isolines is different from the circle and we 
can see the effect of the periodicity of the permittivity distribution in photonic crystals.

The equi-frequency plots are hard to study and analyze. The standard way of representation of $\omega_{n}(k)$ relations is the photonic band diagram. The photonic band diagram for this structure is shown in Figure $8(a)$. Figure $8(\mathrm{~b})$ shows the gap map for this structure for both polarizations. From this gap map, we find that as the radius of the rods is increased, a TM band-gap appears first at $r=$ $0.13099 \mu \mathrm{m}$. At this value of the radius, a TE band-gap also appears resulting in a complete band gap. The complete band gap terminates at a radius of $r=0.4798$ $\mu \mathrm{m}$. Light of any polarization having this band gap frequency cannot propagate through this crystal in any in-plane direction for these values of the radius of the rods. The largest TE band gap for this structure occurs at $r=0.17837 \mu \mathrm{m}$. The largest TM band gap occurs at $r=0.3398 \mu \mathrm{m}$. For this lattice, a large complete band gap is found. The largest complete band gap occurs at $r=0.3372 \mu \mathrm{m}$. Its width is $\Delta \omega=0.06489(2 \pi c / a)$. The band-gap width, mid-gap frequency, gap-midgap ratio and gap percentage for this structure are given in Table 3.

\subsection{Honeycomb Lattice of Air Holes in Germanium}

A two-dimensional honeycomb photonic crystal lattice is considered that is composed of circular air holes in germanium background having $f=0.126$. The band diagram for TE and TM polarizations for this structure is plotted in Figure 9(a).

Figure 9(a) shows that there is no photonic band gap for TE and TM polarizations. However, as we varied the radius of the air holes, photonic band gaps appear as shown in Figure 9(b). At first, a TE band gap appears for this structure at a radius of $r=0.22848 \mu \mathrm{m}$. Then a small TM band gap appears first at $r=$ $0.29907 \mu \mathrm{m}$. Also a complete photonic band gap appears first for $r=0.42312 \mu \mathrm{m}$.

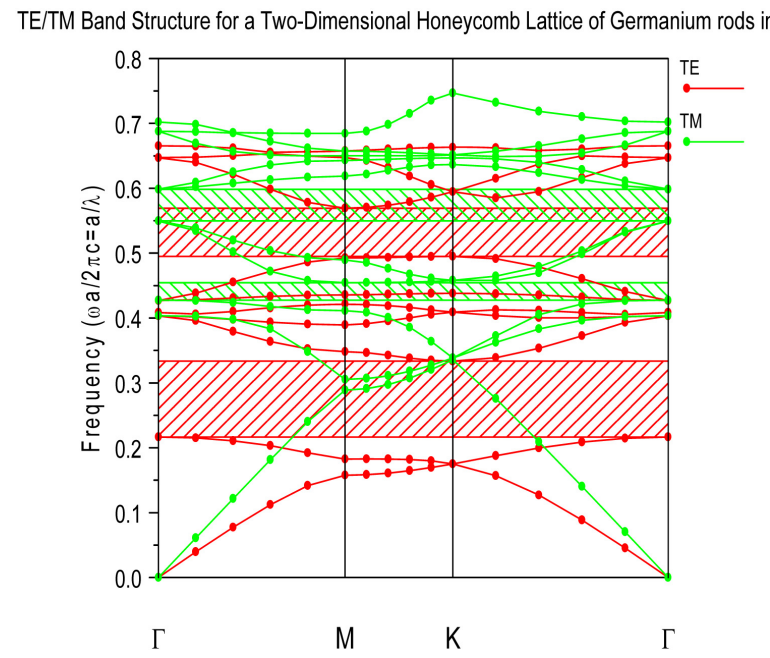

(a)

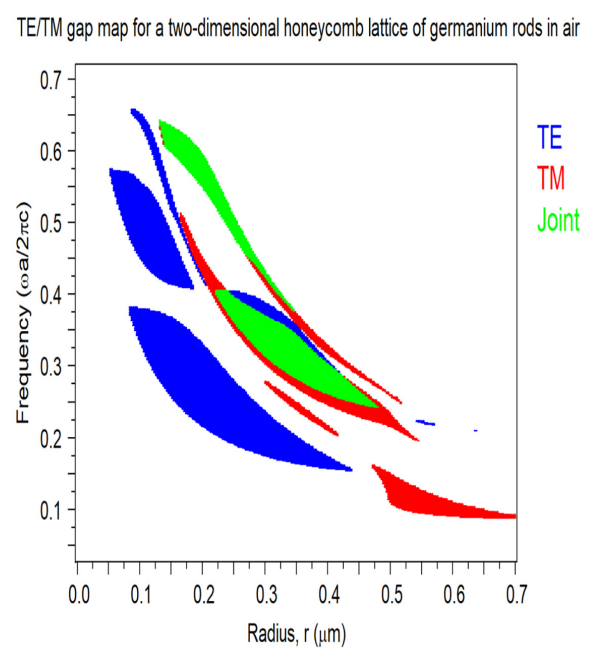

(b)

Figure 8. (a) The TE and TM both or hybrid band diagram for the two-dimensional (2D) honeycomb lattice composed of germanium rods in air and (b) the gap map for this structure. 
Table 3. TE and TM band gap data for the two-dimensional (2D) honeycomb lattice consisting of germanium rods in air.

\begin{tabular}{cccccccc}
\hline $\begin{array}{c}\text { Crystal } \\
\text { structure }\end{array}$ & Polarization & Band & $\frac{\omega a}{2 \pi c}$ & $\begin{array}{c}\text { The mid-gap } \\
\text { frequency }\left(\omega_{m}\right)\end{array}$ & $\begin{array}{c}\text { Band-gap width } \\
(\Delta \omega)\end{array}$ & $\begin{array}{c}\text { Gap-midgap ratio } \\
\left(\Delta \omega / \omega_{m}\right)\end{array}$ & $\begin{array}{c}\text { Gap } \\
\text { percentage }(\%)\end{array}$ \\
\hline & TE & $1^{\text {st }}$ & $0.2166-0.3334$ & 0.2750772504169 & 0.1168560948505 & 0.4248119198277 & 42.48 \\
$\begin{array}{c}\text { Honeycomb } \\
\text { lattice of } \\
\text { germanium } \\
\text { rods in air }\end{array}$ & TM & $2^{\text {nd }}$ & $0.49488-0.56921$ & 0.4242457602636 & 0.005377592663194 & 0.01267565445993 & 1.26 \\
& $\begin{array}{c}\text { TE and TM } \\
\text { both or hybrid }\end{array}$ & $1^{\text {st }}$ & $0.42753-0.4545$ & 4.409849258957 & 0.02702520774712 & 0.06128374499930 & 6.13 \\
& $2^{\text {st }}$ & $0.54962-0.56921$ & 0.5740363729760 & 0.04867417560238 & 0.08479284222016 & 8.48 \\
\hline
\end{tabular}

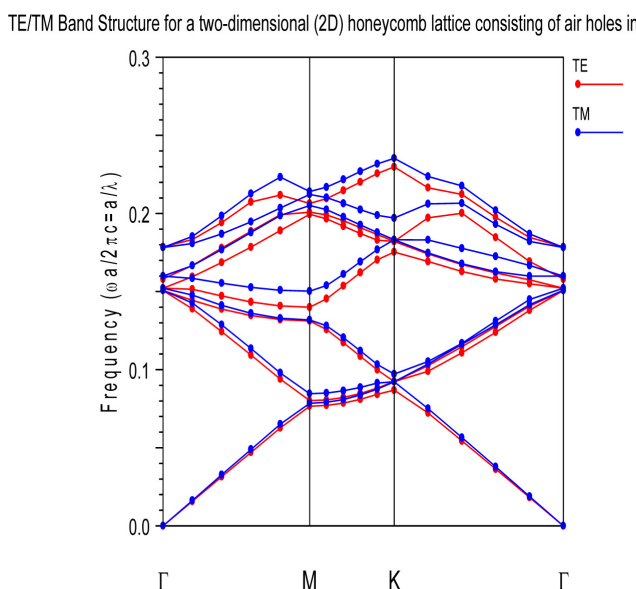

(a)
TE/TM gap map for the 2D honeycomb lattice of air holes in germanium

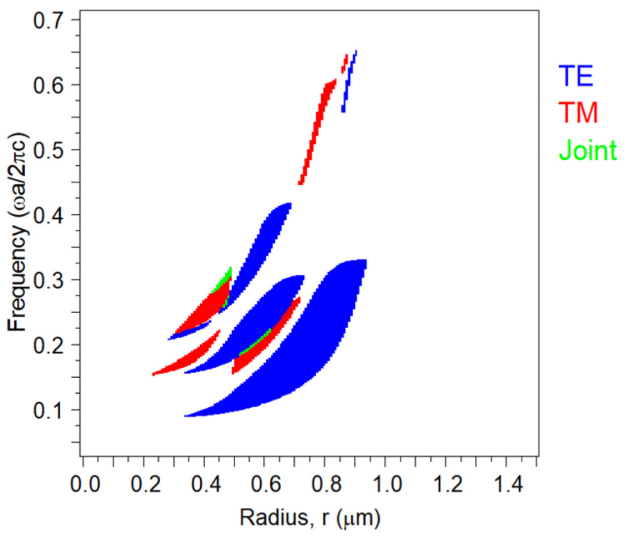

(b)

Figure 9. (a) The TE and TM both or hybrid band diagram for the two-dimensional (2D) honeycomb lattice consisting of air holes in germanium and (b) the gap map for this structure.

The largest TE gap occurs at $r=0.7002 \mu \mathrm{m}$. The largest TM gap occurs at $r=$ $0.47339 \mu \mathrm{m}$. The largest complete gap occurs at $r=0.48563 \mu \mathrm{m}$. Its width is $\Delta \omega$ $=0.014(2 \pi c / a)$.

\section{Conclusion}

We considered Ge rods inserted into air background as well as air holes drilled in Ge background. We observed that TE modes have advantage in photonic crystal structures composed of Ge rods in air background whereas TM modes are favoured in photonic crystal structures composed of air holes in Ge background. Firstly, we considered a rod or hole radius of $r=0.2 \mu \mathrm{m}$. For this radius value, we observed a larger and wider TE band gap for the hexagonal lattice of Ge in air in comparison to that of the honeycomb lattice. This is because of the difference in structure. The unit cell of a hexagonal lattice contains only one lattice site whereas that of a honeycomb lattice contains two lattice sites. This makes the hexagonal lattice easier to create larger gap. We did not find a photonic band gap for two-dimensional honeycomb lattice of air holes in Ge. But for hexagonal lattice of air in Ge, we found a TM band gap. Then, we investigated the effect of the variation of radius of Ge rods or air holes on the photonic band 
gap. Photonic band gap appears for almost all structures with the variation of the radius, as shown in the gap maps. For photonic crystal structures composed of Ge rods in air, it was found that, with the increase of the radius $r$, the gap width decreases and the gaps are decreased in frequency. This is because in this case the frequency is inversely proportional to the refractive index of a medium and the average refractive index of the medium steadily increases with the increase of the radius [14]. On the other hand, for photonic crystal structures of air holes in Ge background, the gap width increases and the gaps are increased in frequency with the increase of the radius. We have shown the actual view of photonic band gaps in the surface plots. The projection of different surfaces on the wave-vector plane is shown as the equifrequency plots. Nowadays, $\mathrm{Si}$ is used to fabricate photonic devices. But, according to our simulation result, Ge based 2D PCs provide larger photonic band gap than that of $\mathrm{Si}$ [18]. That is why, we may say that, Ge is a very good candidate for fabricating useful photonic devices.

\section{Acknowledgements}

The authors thank the Information and Communication Technology Division (ICT-Division), Department of the Ministry of Posts, Telecommunications and Information Technology of the Government of Bangladesh, for supporting this research work.

\section{Funding Information}

This work is supported by the Higher Education Quality Enhancement Program (HEQEP) project of University Grant Comission (UGC) of the Government of Bangladesh.

\section{Conflicts of Interest}

The authors declare no conflicts of interest regarding the publication of this paper.

\section{References}

[1] Joannopoulos, J.D., Johnson, S.G., Winn, J.N. and Meade, R.D. (2008) Photonic Crystals: Molding the Flow of Light. 2nd Edition, Princeton University Press, Princeton.

[2] Sakoda, K. (2004) Optical Properties of Photonic Crystals. 2nd Edition, Springer, Berlin.

[3] Yablonovitch, E. (1987) Inhibited Spontaneous Emission in Solid-State Physics and Electronics. Physical Review Letters, 58, 2059-2062.

https://doi.org/10.1103/PhysRevLett.58.2059

[4] Krauss, T.F., La Rue, R.M.D. and Brand, S. (1996) Two-Dimensional Photonic-Bandgap Structures Operating at Near-Infrared Wavelengths. Nature, 383, 699-702. https://doi.org/10.1038/383699a0

[5] Skorobogatiy, M. and Yang, J. (2008) Fundamentals of Photonic Crystal Guiding. Cambridge University Press, Cambridge. 


\section{https://doi.org/10.1017/CBO9780511575228}

[6] Sukhoivanov, I.A. and Guryev, I.V. (2009) Photonic Crystals Physics and Practical Modeling. Springer Series in Optical Sciences, Berlin.

[7] John, S. (1987) Strong Localization of Photons in Certain Disordered Dielectric Superlattices. Physical Review Letters, 58, 2486-2489. https://doi.org/10.1103/PhysRevLett.58.2486

[8] Yablonovitch, E. (1993) Photonic Band-Gap Crystals. Journal of Physics. Condensed Matter, 5, 2443-2460. https://doi.org/10.1088/0953-8984/5/16/004

[9] Li, Z.-Y., Gu, B.-Y. and Yang, G.-Z. (1998) Large Absolute Band Gap in 2D Anisotropic Photonic Crystals. Physical Review Letters, 81, 2574-2577. https://doi.org/10.1103/PhysRevLett.81.2574

[10] Plihal, M. and Maradudin, A.A. (1991) Photonic Band Structure of Two-Dimensional Systems: The Triangular Lattice. Physical Review B, 44, 8565-8571. https://doi.org/10.1103/PhysRevB.44.8565

[11] Wen, F., David, S., Checoury, X., El Kurdi, M. and Boucaud, P. (2008) Two-Dimensional Photonic Crystals with Large Complete Photonic Band Gaps in Both TE and TM Polarizations. Optics Express, 16, 12278-12289. https://doi.org/10.1364/OE.16.012278

[12] Ivinskaya, A.M., Lavrinenko, A.V., Shyroki, D.M. and Sukhorukov, A. (2013) Single and Coupled Nanobeam Cavities. In: Passaro, V.M.N., Ed., Advances in Photonic Crystals, InTechOpen, London, 27-50.

[13] Qiu, M. and He, S. (2000) Guided Modes in a Two-Dimensional Metallic Photonic Crystal Waveguide. Physics Letters A, 266, 425-429.

https://doi.org/10.1016/S0375-9601(00)00049-9

[14] Winn, J.N., Meade, R.D. and Joannopoulos, J.D. (1994) Two-Dimensional Photonic Band-Gap Materials. Journal of Modern Optics, 41, 257-273. https://doi.org/10.1080/09500349414550311

[15] Joannopoulos, J.D., Winn, J.N. and Meade, R.D. (1995) Photonic Crystals: Molding the Flow of Light. Princeton University Press, Princeton.

[16] Sukhoivanov, I.A. and Guryev, I.V. (2005) Photonic Crystals Physics and Practical Modeling. Springer, Berlin.

[17] McCall, S., Platzman, P., Dalichaouch, R., Smith, D. and Schultz, S. (1991) Microwave Propagation in Two-Dimensional Dielectric Lattices. Physical Review Letters, 67, 2017-2020. https://doi.org/10.1103/PhysRevLett.67.2017

[18] Jamois, C., Wehrspohn, R.B., Andreani, L.C., Hermann, C. and Gösele, U. (2003) Silicon-Based Two-Dimensional Photonic Crystal Waveguides. Photonics and $\mathrm{Na}$ nostructures-Fundamental and Applications, 1, 1-13.

[19] Griffiths, D.J. (1999) Introduction to Electrodynamics. 3rd Edition, Prentice-Hall, Upper Saddle River.

[20] Taflove, A. and Hagness, S.C. (2005) Computational Electrodynamics: The Finite-Difference Time-Domain Method. 3rd Edition, Artech House, Norwood.

[21] Shi, S., Chen, C. and Prather, D.W. (2004) Plane-Wave Expansion Method for Calculating Band Structure of Photonic Crystal Slabs with Perfectly Matched Layers. Journal of the Optical Society of America, 21, 1769.

https://doi.org/10.1364/JOSAA.21.001769

[22] Antos, R. and Veis, M. (2012) Fourier Factorization in the Plane Wave Expansion Method in Modeling Photonic Crystals. In: Photonic Crystals-Introduction, Applications and Theory, IntechOpen, London, 319-344. 
[23] He, J.-P., He, S.-L., Shen, L.-F. and Xiao, S.-S. (2002) Large Absolute Photonic Bandgap at High Frequencies in a Two-Dimensional Photonic Crystal with a Hexagonal Structure. Chinese Physics Letters, 19, 69-72. https://doi.org/10.1088/0256-307X/19/1/322

[24] Zabelin, V. (2009) Numerical Investigations of Two-Dimensional Photonic Crystal Optical Properties, Design and Analysis of Photonic Crystal Based Structures. EPFL, Lausanne, 131. 